SUPPORTING INFORMATION

\title{
Directed evolution of stabilized monomeric CD19 for monovalent CAR interaction studies and monitoring of CAR-T cell patients
}

Elisabeth Laurent, Anna Sieber, Benjamin Salzer, Anna Wachernig, Jacqueline Seigner, Manfred Lehner, René Geyeregger, Bernhard Kratzer, Ulrich Jäger, Renate Kunert, Winfried F. Pickl, Michael W. Traxlmayr ${ }^{*}$

*Corresponding Author:

Department of Chemistry, BOKU - University of Natural Resources and Life Sciences, Muthgasse 18, 1190 Vienna, Austria; orcid.org/0000-0002-2108-582X; Phone: +43 147654 77274; Email: michael.traxlmayr@boku.ac.at

\section{CONTENTS}

Figure S1. Effect of thermal denaturation on binding of different mAbs.

Figure S2. Characterization of libraries M and XL by sequencing of 48 clones, respectively.

Figure S3. One hot spot mutation is sufficient to improve the structural integrity and thermal stability of CD19-ECD. 


\section{SUPPLEMENTARY FIGURES}
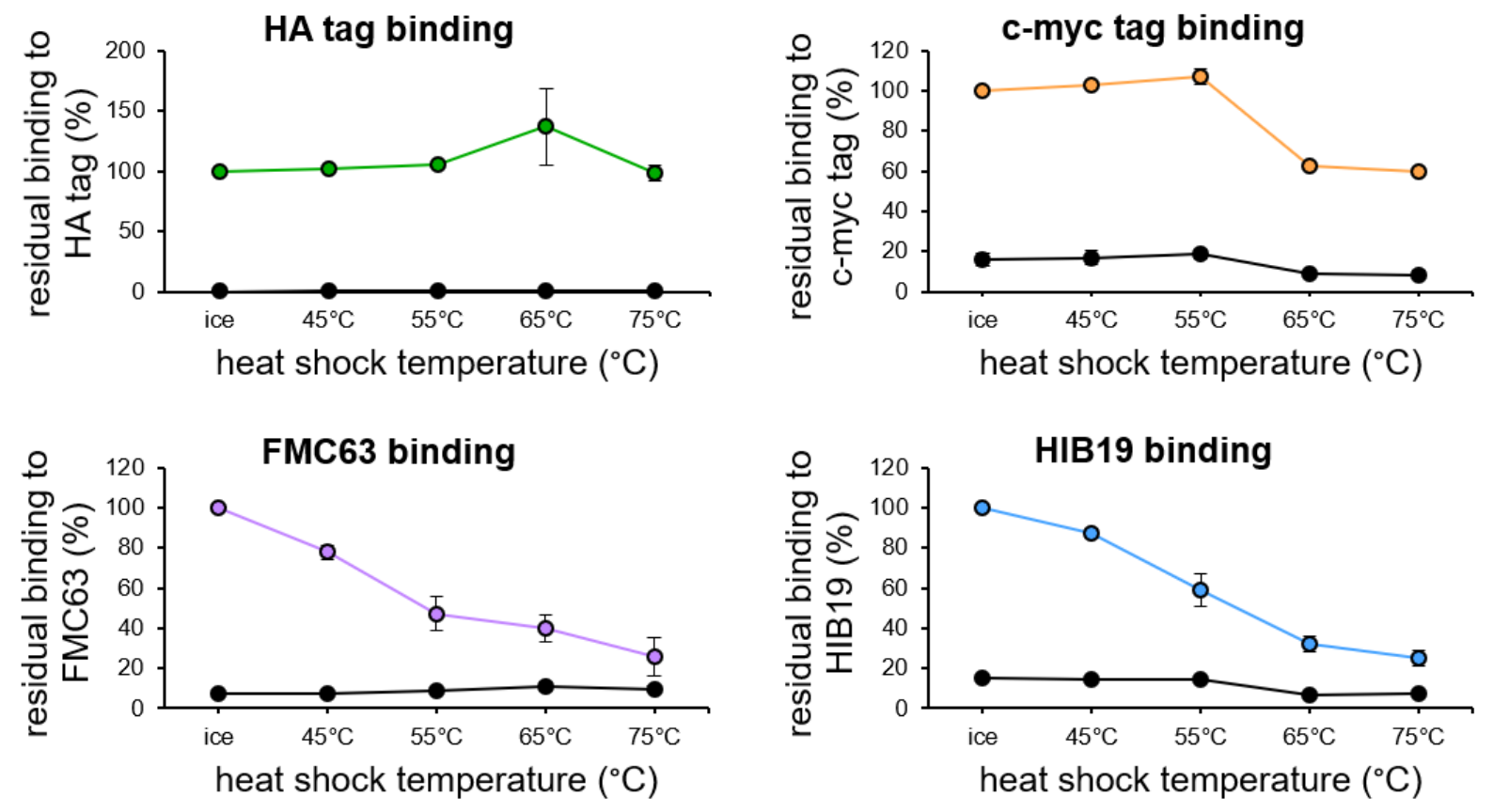

Figure S1. Effect of thermal denaturation on binding of different mAbs. Flow cytometric analysis of yeast cells displaying CD19-wt after incubation at increasing temperatures for $10 \mathrm{~min}$ and staining with anti-HA mAb, anti-c-myc mAb, FMC63 and HIB19, respectively. The black curve represents the negative control [staining with secondary antibody only (for FMC63 and antic-myc) or cellular autofluorescence (for anti-HA and HIB19, which were both directly labeled]. The residual binding of the mAbs is plotted versus the incubation temperature (normalized to the mean of non-heated yeast cells binding to the respective mAb). Averages \pm SDs of three independent experiments are shown. 


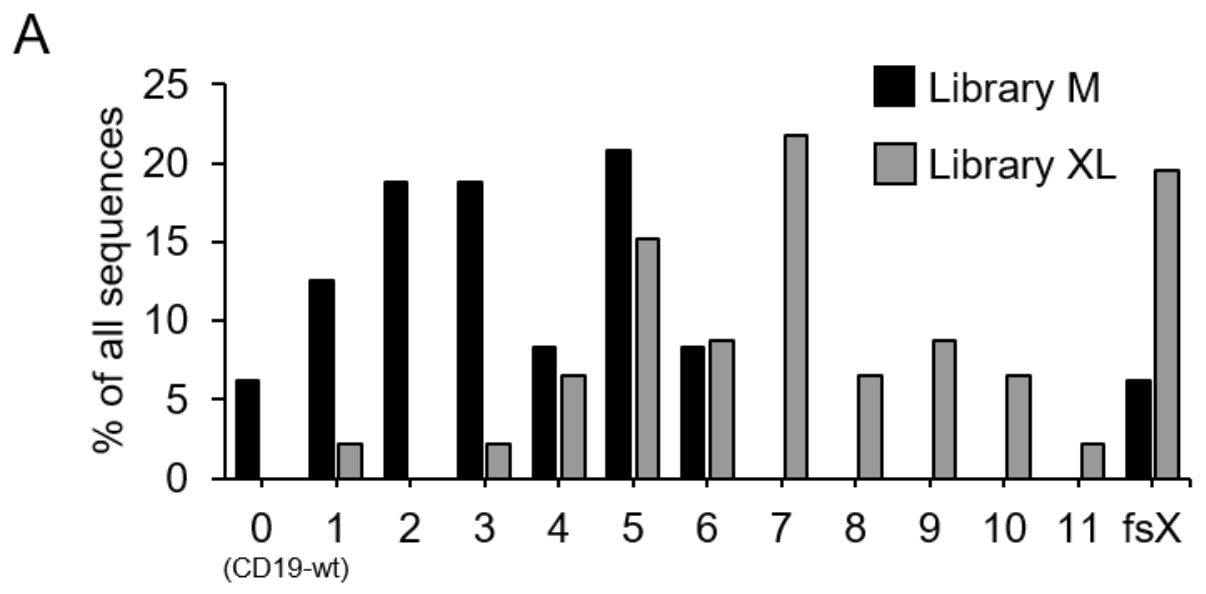

number of point mutations per CD19-gene

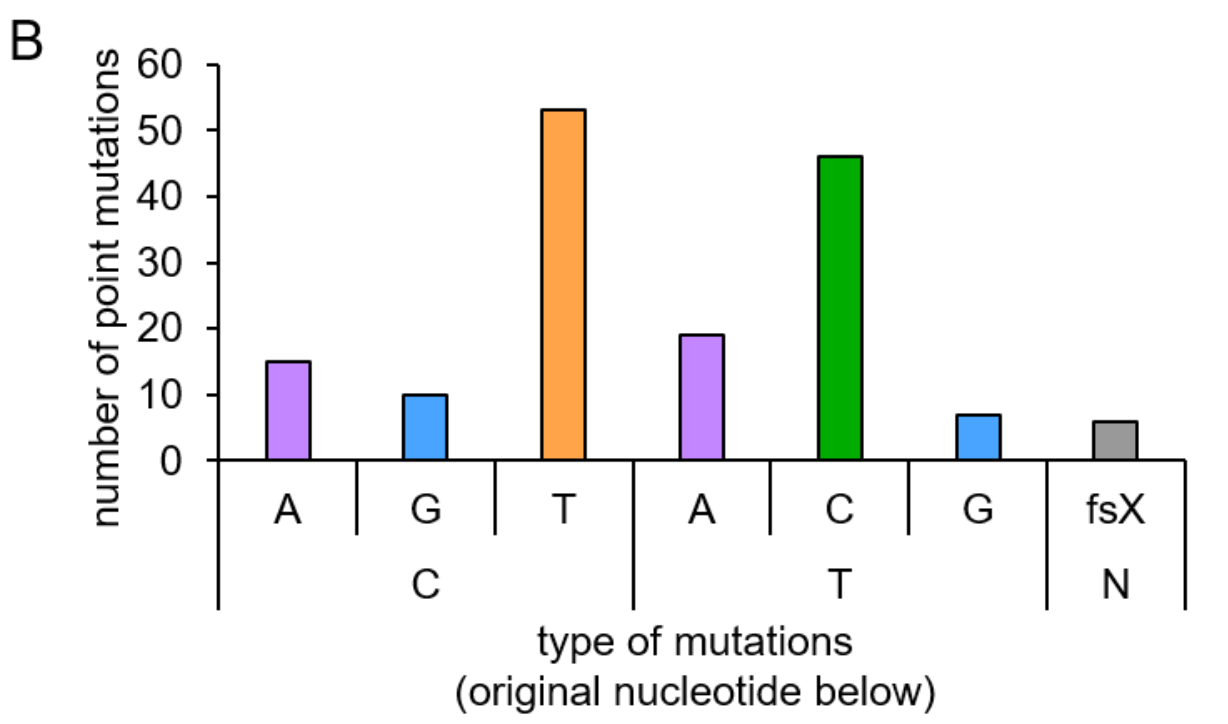

Figure S2. Characterization of libraries $M$ and XL by sequencing of 48 clones, respectively. (A) The frequency of detected nucleotide mutations over the CD19-wt gene (P20-P278) for both libraries is shown. (B) The number of each possible nucleotide mutation found in library $\mathrm{M}$ is shown. fsX refers to frameshift mutations. 
A

FMC63 binding
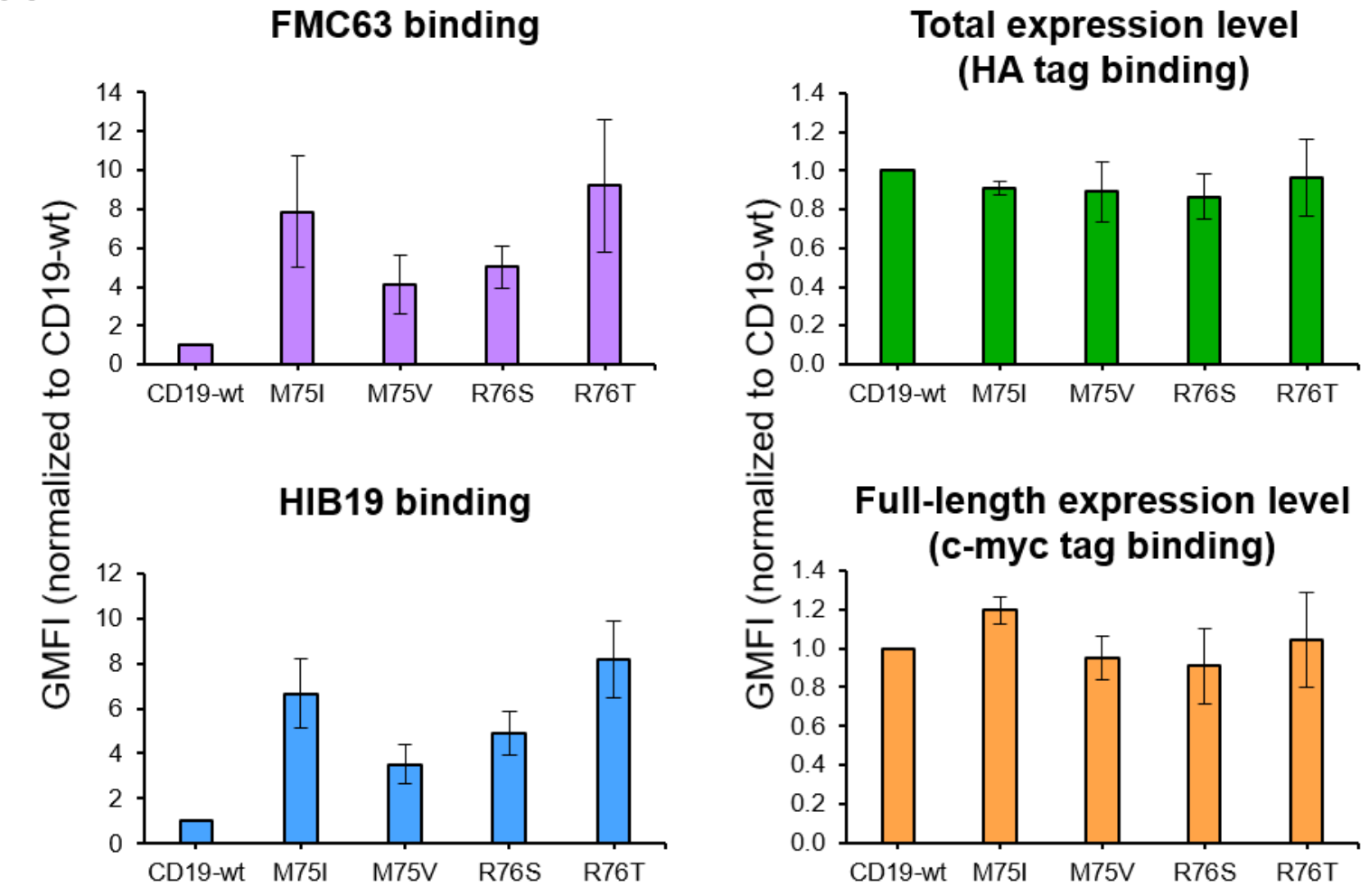

B

$T_{1 / 2}$ values

C
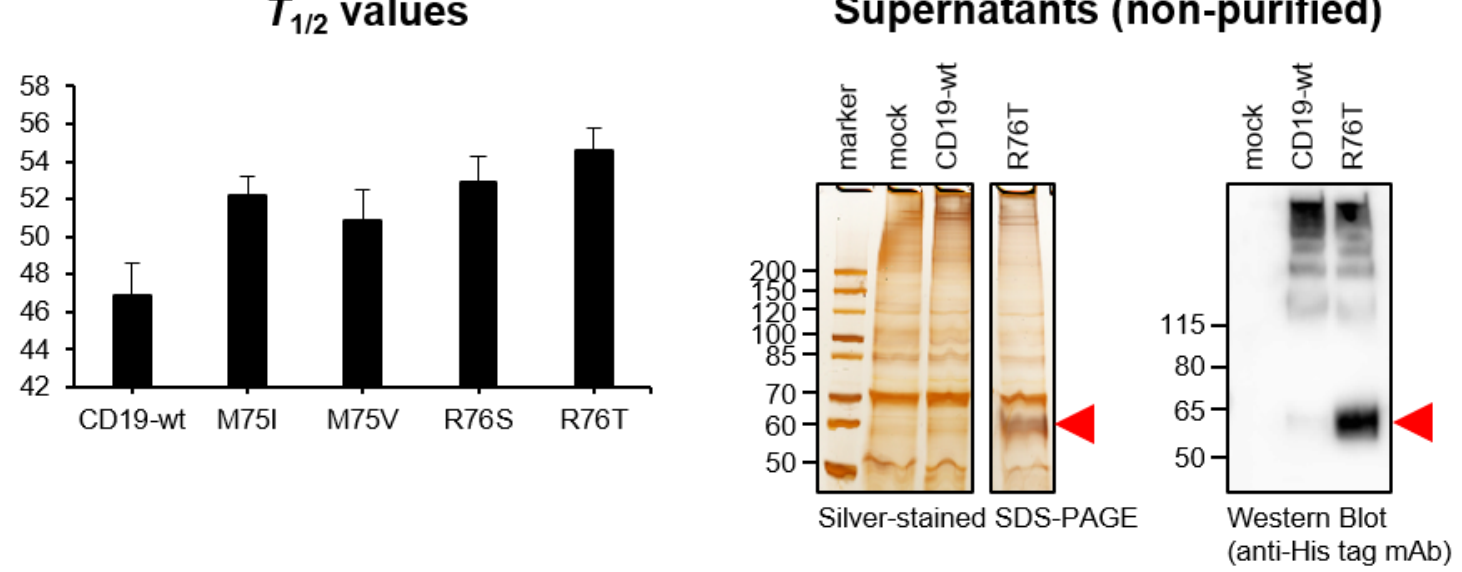

Figure S3. One hot spot mutation is sufficient to improve the structural integrity and thermal stability of CD19-ECD. (A) Analysis of binding properties of single SF mutants (M75I, M75V, R76S, R76T) relative to CD19-wt. Displaying yeast cells were probed for binding to the structure specific anti-CD19-ECD mAbs (FMC63 and HIB19) and the anti-tag mAbs (anti-HA and anti-cmyc) by flow cytometry. (B) Determination of the $T_{1 / 2}$ values of single SF mutants relative to 
CD19-wt. Displaying yeast cells were incubated at increasing temperatures for $10 \mathrm{~min}$ and subsequently analyzed for their binding to HIB19 by flow cytometry. Resulting data allow the

calculation of $T_{1 / 2}$ values based on modelled denaturation curves. ${ }^{1,2}$ The plot shows calculated $T_{1 / 2}$ values of CD19-wt and single SF variants. All data in this figure represent averages \pm SDs of three independent experiments. (C) Analysis of the supernatant of HEK293-6E cells transiently transfected with plasmids encoding CD19-wt or the R76T single SF mutant by silver-stained SDSPAGE and Western Blot detecting His-tagged protein. Mock (negative control) refers to cells that were transfected with sterile $\mathrm{H}_{2} \mathrm{O}$ instead of plasmid DNA. The red marks indicate migration of the monomeric CD19-ECD protein.

\section{REFERENCES}

[1] Orr, B. A., Carr, L. M., Wittrup, K. D., Roy, E. J., and Kranz, D. M. (2003) Rapid method for measuring ScFv thermal stability by yeast surface display, Biotechnology progress 19, 631-638.

[2] TraxImayr, M. W., and Shusta, E. V. (2017) Directed Evolution of Protein Thermal Stability Using Yeast Surface Display, Methods Mol Biol 1575, 45-65. 